Classification

Physics Abstracts

$61.72 \mathrm{~F}-81.40$

\title{
Lattice Electron Microscopy and Image Processing of Ion-Implanted and Laser-Annealed GaAs Structures
}

\author{
Gianfranco Vitali $\left({ }^{1}\right)$, Marco Rossi $\left({ }^{1}\right)$, Giuseppe Zollo $\left({ }^{1}\right)$, Cesare Pizzuto $\left({ }^{1}\right)$, \\ Nikolai Pashov $\left({ }^{2}\right)$ and Maria Kalitzova $\left({ }^{2}\right)$ \\ $\left({ }^{1}\right)$ Dipartimento di Energetica, Università “La Sapienza”, via A. Scarpa, 14 - 00161 Roma and INFM, \\ UdR RM I, Italy \\ $\left({ }^{2}\right)$ Institute of Solid State Physics, Bulgarian Academy of Sciences, Tzarigradsko Chaussee, 72 - 1784 \\ Sofia, Bulgaria
}

(Received March 1; accepted May 22, 1995)

\begin{abstract}
The efficiency of the Low-Power Pulsed-Laser Annealing (LPPLA) as a treatment to restore the ion implantation induced crystal disorder is well-known, but it is still open the question regarding the way in which is possible to model the LPPLA dynamic effects. At this purpose we believe that a deeper knowledge about the modification of the ion-induced extended defects (dislocations, stacking faults, clusters), as a consequence of the LPPLA treatment, is relevant. In our opinion, a technique suitable to gain this information could be the lattice image digital processing. In this paper we reported the experimental results concerning the use of this technique applied to some HRTEM images of low-dose implanted GaAs samples. In particular, the appearance of different types of extended defects in the implanted material and their annealing with LPPLA have been put in evidence.
\end{abstract}

\section{Introduction}

The main advantages of ion implantation as a technique to develop GaAs devices and integrated circuits are well-established. The active channel formation in similar implanted substrates is believed to be a key process in the high-speed electronic technology [1].

The nature of the damage created in as-implanted substrates is important for the efficiency of the next stages in the processing, because the extended defect structure, their diffusion and annealing affect in a strong way the electrical devices properties. In the ion implantation doping of materials as GaAs, a large number of atoms (several thousand) are displaced in collision cascades, creating defects zones in the crystal lattice characterized by a vacancies core with a periphery rich of interstitials [2]. What kind of point-defect clustering in such zinc-blend structure preferentially develops, far from the thermodynamic equilibrium, is still an open question. For the case of FCC metals, it seems now well-established that the microscopical processes occurring during the ion implantation mainly induce the formation of edge dislocations and dislocation loops of interstitial and vacancy type in the cascade-volume [3]. Transmission electron microscopy (TEM) has been 
extensively used in the past to obtain direct information about the defect-zones produced by individual displacement cascades and about the in-depth radiation-damage distribution. In this way TEM provides knowledge on the processes generating structural damage in the ion implantation of semiconducting materials.

The possibility to perform the annealing of ion implanted GaAs, with the Low-Power PulsedLaser treatment (LPPLA) at temperatures far below the dissociation temperature of the material [4-6], provides new aspects of interest for the Electron Microscopy, connected with the study of very fine crystal structural details into the atomic structure of the damaged zones and their evolution during the annealing. Comprehensive information about such characteristic structures can been gained by additional digital processing of the obtained images [7]. This technique can furnish a valid complementary investigation to the electrical measurements to evaluate the effect of the LPPLA.

The damage produced by the ion implantation or the residual one detectable after the LPPLA, together with the electron microscopy instabilities, give pictures characterized by particulars with a ratio between the signal and the noise $(\mathrm{S} / \mathrm{N})$ that can be very low. In the HRTEM of examined specimens, our interest is exactly directed to casual imperfections of the periodic structure as defect clusters, dislocation loops, grain boundaries, interfaces etc.. As a consequence, the physical situation is particularly suitable for the image processing to improve the $\mathrm{S} / \mathrm{N}$ ratio.

\section{Experimental}

Single crystal semi-insulating (100) GaAs wafers were implanted with $140 \mathrm{keV} \mathrm{Zn}{ }^{+}$ions, a fluence of $D=10^{14} \mathrm{~cm}^{-2}$, at temperature $T=110 \pm 10^{\circ} \mathrm{C}$ and random direction.

The laser annealing has been performed in air and at room temperature by 30 successive superimposed pulses, at a frequency of $0.05 \mathrm{~Hz}$, each of them with a density power $P_{0}=4.5 \mathrm{MWcm}^{-2}$ from a Q-switched ruby-laser $(\lambda=694 \mathrm{~nm})$ with a pulse duration $\Delta t_{\mathrm{FWHM}}=25 \mathrm{~ns}$.

The above mentioned value of $P_{0}$ is lower than the optimal requested for annealing (ranging between 5 and $6 \mathrm{MW} \mathrm{cm}^{-2}$ ) as already shown [4-6]. The calculated maximum temperature of the material (as described elsewhere [8]) in the present irradiation conditions does not exceed $700 \mathrm{~K}$.

The used annealing conditions, were expected to be characterized by well expressed stages of the defects evolution.

All the following reported HRTEM investigations, performed on as-implanted and LPPLA treated samples, have been carried out by a 4000Ex-Jeol instrument operating at $400 \mathrm{kV}$ in bright field many-beam mode.

The obtained HRTEM micrographs have been successively digitalized and processed in a Kontron System in order to improve the signal to noise ratio $\mathrm{S} / \mathrm{N}$ with a negligible loss of resolution.

At this regard, one can suppose that a high resolution electron-microscopy image is obtained as a resultant of three superimposed contributions:

i) a known periodic signal $P(r)$ connected with the periodic crystal lattice potential;

ii) an unknown signal $X(r)$, that is the subject under study, not periodic but connected with the periodic structure;

iii) the noise $N(r)$ with a casual intensity and linked with the specimen or/and with the electronmicroscopy instabilities. In a first approximation, the above mentioned contributions are additive, so that the intensity in any point of the picture is given by:

$$
S(r)=P(r)+X(r)+N(r) .
$$

The filtered image in the spatial frequencies field will be:

$$
\operatorname{Sp}(g)=P(g) F(g)+X(g) F(g)+N(g) F(g)
$$


where the filtering function $F(g)$ will be chosen in order to minimize the product $N(g) F(g)$ and, in the same time, to give maximum $X(g) F(g)$. This choice is the criterion for the filter selection: this requirement corresponds to find the best balance between the contrast and/or the image resolution of the object under study. So the $\mathrm{S} / \mathrm{N}$ ratio of the examined micrographs showing a periodic motif, can be increased through the convolution provided by an optical filter consistent of circular apertures in the position of the optical diffraction spots (Bragg Filters).

\section{Results and Discussions}

A typical appearance of the as-implanted planar specimens as seen by HRTEM is shown in Figure 1a. In this case it was possible to observe resolved one set of (220) lattice fringes $\left(d_{220}=\right.$ $1.99 \AA$ A). Isolated and overlapped damaged zones are characterized by a specific contrast and appear in the micrographs as dark regions. In a first approximation, the atomic rows in a damaged zone (like $C_{1}$ in the Fig. 1a) are coherent with the adjacent rows of the undisturbed matrix. This circumstance confirms that, in the present implantation conditions, the deposited energy density (with an estimated mean value of about $0.01 \mathrm{eV} / \mathrm{at}$ ) is not enough for the appearance of the

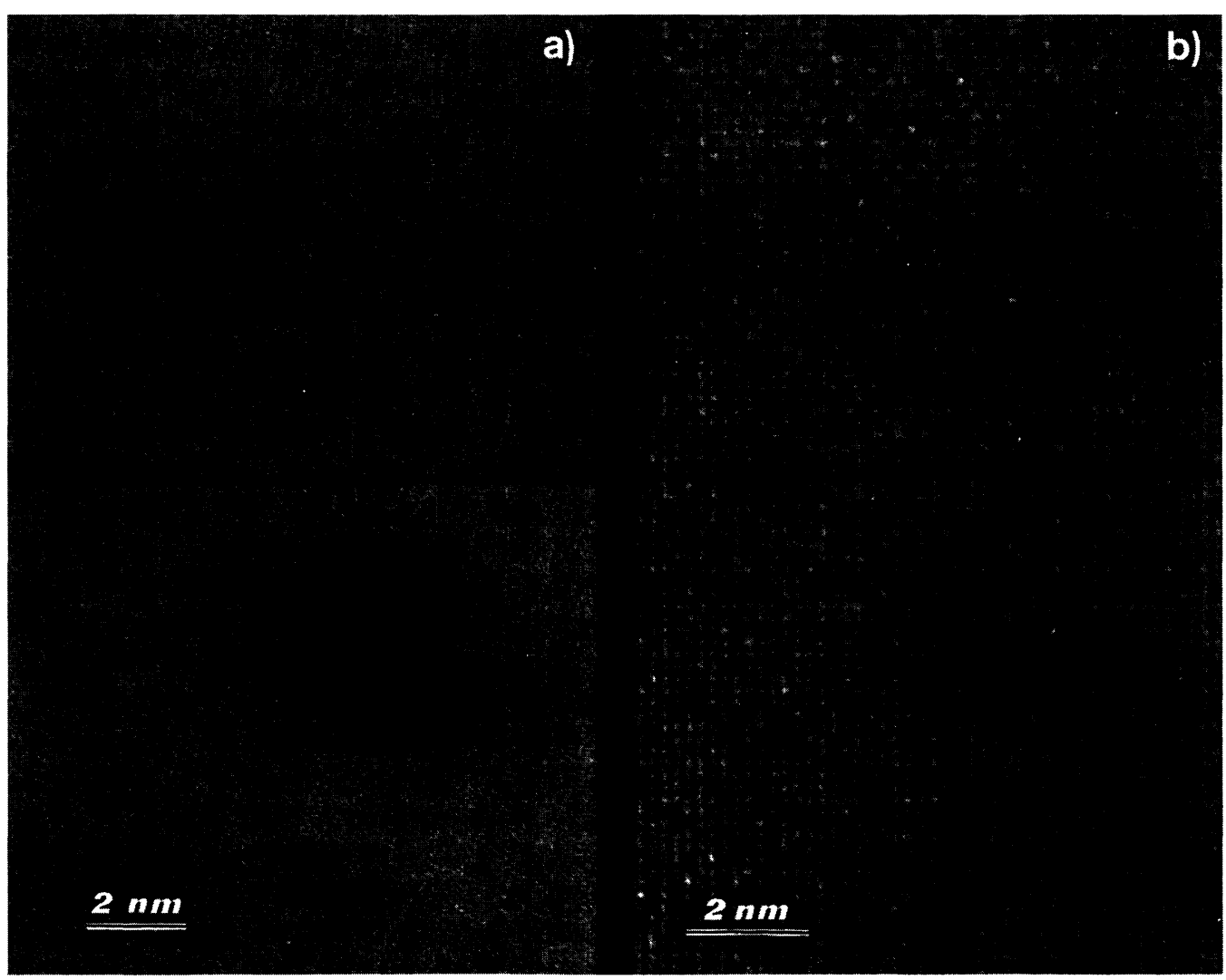

Fig. 1. - HRTEM micrographs of planar (100) GaAs specimens: a) after ion implantation (an isolated damage zone is labelled $\mathrm{C}_{1}$ ); b) after ion implantation and low-power pulsed laser annealing (a residual damage zone is labelled $\mathrm{C}_{2}$ ). 


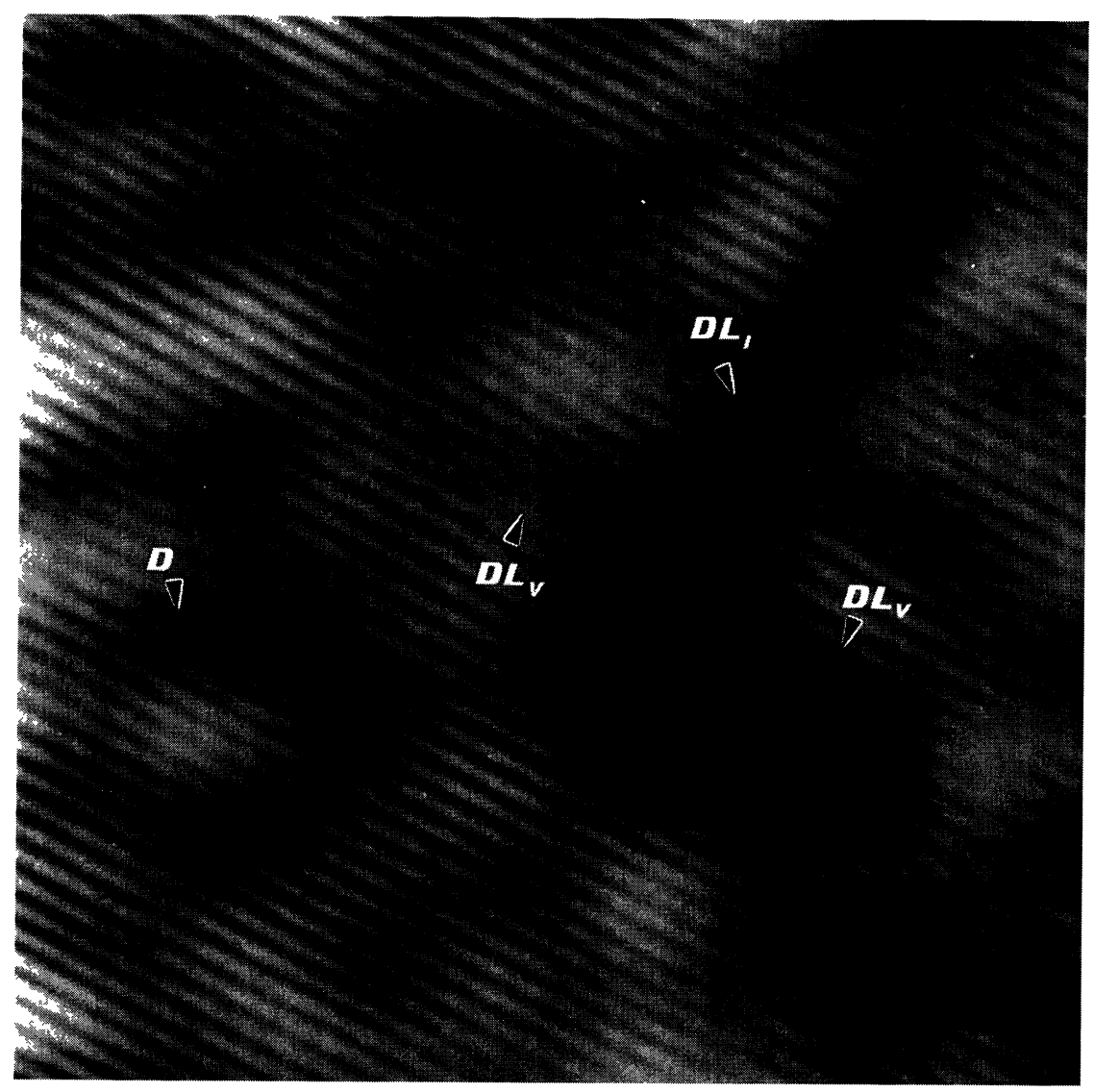

Fig. 2. - Filtered image, with one pair of reflections corresponding to one set of (220) planes, of the isolated damage zone $\mathrm{C}_{1}$ shown in Figure 1a. Edge dislocations (D), dislocation loops of interstitial $\left(\mathrm{DL}_{\mathrm{i}}\right)$ and vacancy $\left(\mathrm{DL}_{\mathrm{v}}\right)$ type are indicated.

amorphization within the displacement cascade, the reported critical energy density for the amorphization being around $2.0 \mathrm{eV} /$ at for irradiation at $300 \mathrm{~K}$ [9]. The ion-induced damage produced in such conditions is classified as "type I defects", i.e. defects forming with a dose exceeding a critical dose necessary for the formation of extended defects but not sufficient to obtain an amorphous layer [10]. In the present case, the distribution of type I detects has a maximum in correspondence with the measured projected range $\left(R_{\mathrm{p}}\right.$ about $\left.85 \mathrm{~nm}\right)$, higher than the calculated one due to the temperature enhanced diffusion of impurity and point defects [9].

The main source of point defects for the damaged zones formation is the Frenkel pairs production during the collision cascades phenomena. When the displacement events in the thermal spikes stop (i.e. the colliding atoms loose their energy) a spatial arrangement of Frenkel defects forms with vacancies at the center and self-interstitials in the periphery that can organize themselves in small edge dislocations or dislocation loops [11-13]. Moreover, the vacancy-rich core can relax too and form vacancy clusters such as dislocation loops. In the present case, the elevated temperature implantation conditions can enhance the diffusion and the clustering of Frenkel pairs out of the collision cascade. The inner structure of the so formed damaged zones, as those named 


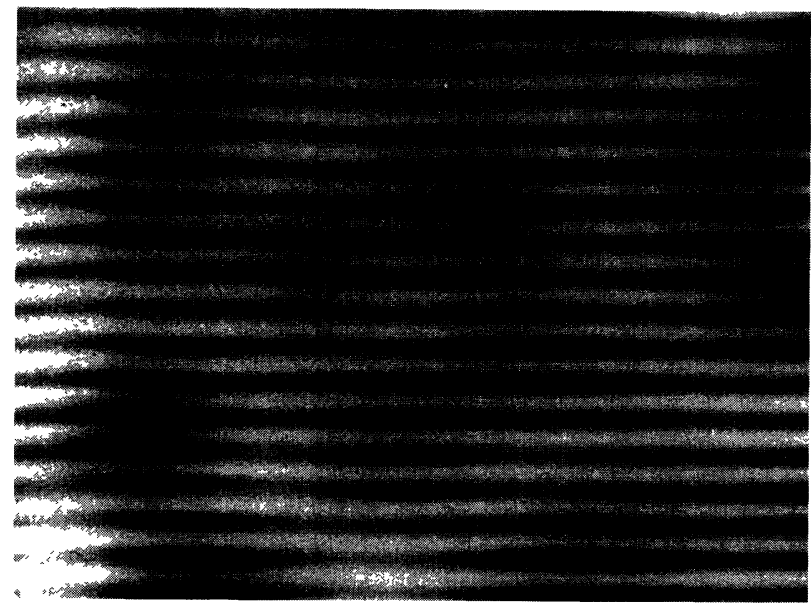

Fig. 3. - Filtered image, with one pair of reflections corresponding to one set of (220) planes, of the isolated damage zone $\mathrm{C}_{2}$ shown in Figure $1 \mathrm{~b}$.

$\mathrm{C}_{1}$ in Figure 1a, and their evolution under the LPPLA treatment could in principle be viewed through High Resolution Transmission Electron Microscopy but a poor S/N ratio can inhibit this possibility. In order to obtain more precise information about the kind of localized periodicity disturbances in the images of the isolated damaged zones, they were digitally processed using Bragg filters in the Fourier space with one or two pairs of strong reflections.

From the experimental results, it was concluded that an useful filter configuration was one using a pair of strong reflections corresponding to one set of $(220)$ resolved lattice planes. In this case the reconstructed image of the analysed part of the crystal lattice projection was presented with an improved contrast for all periodicity disturbances.

The filtered image of an isolated damaged zone of an as-implanted GaAs crystal, observed by HRTEM in $<100>$ projection, is shown in the Figure 2. It clearly shows that the damaged regions contain a large number of extended lattice defects, mainly dislocation loops of vacancy and interstitial type (DL $\mathrm{L}_{v}$ or $\mathrm{DL}_{\mathrm{i}}$ in the Fig. 2) and edge dislocations (indicated with $\mathrm{D}$ ). The distribution of the observable extended defects is not homogeneous with a calculated average density of the order of $10^{13} \mathrm{~cm}^{-2}$.

The effects of LPPLA treatment to recover the lattice order of the ion-implanted specimen, is clearly visible in the obtained filtered image of residual damaged zones of an implanted and LPPLA treated sample $\left(\mathrm{C}_{2}\right.$ in the Fig. $\left.1 \mathrm{~b}\right)$ shown in Figure 3 ; the same filtering procedure described above has been applied.

Moreover, a filtered image of the same damaged zone, generated using a Bragg filter with two pairs of strong reflections, is shown in Figure 4.

From the filtered images of the damaged zones in LPPLA specimens, is clear that no extended defects (as dislocation loops) are found in the examined zone and only very weak disturbances can be detected: it is worth noting the efficiency of the applied LPPLA treatment in the recovering of the GaAs lattice crystallinity, compromised by ion implantation.

Only as a reference, a filtered image of an undisturbed part of an implanted sample is shown in the Figure 5. This picture was obtained in the same conditions of all above: the similarity with that shown in the Figure 3 is evident. 


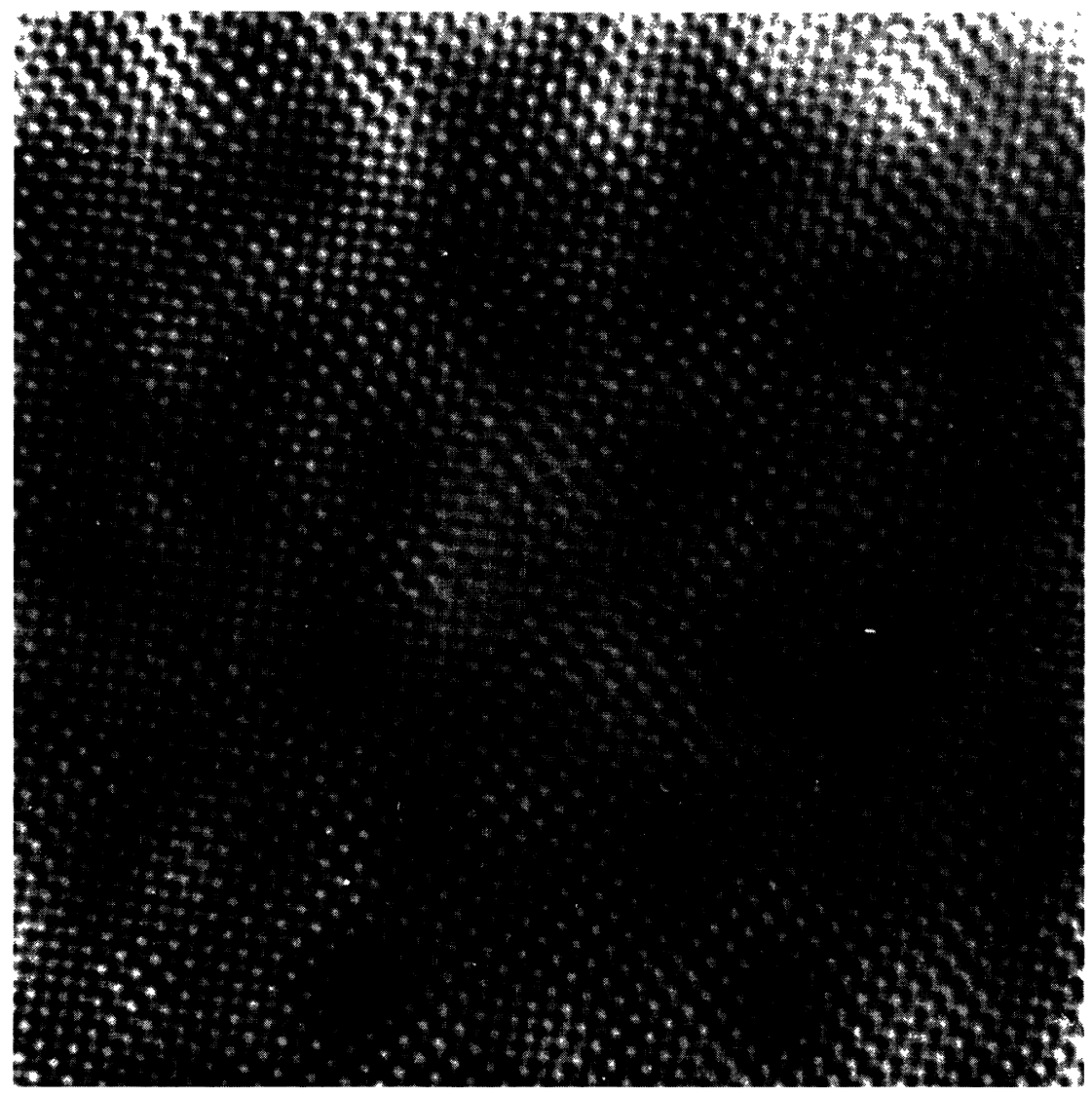

Fig. 4. - Filtered image, with two perpendicular pairs of reflections corresponding to two sets of (220) planes, of the isolated damage zone $\mathrm{C}_{2}$ shown in Figure $1 \mathrm{~b}$.

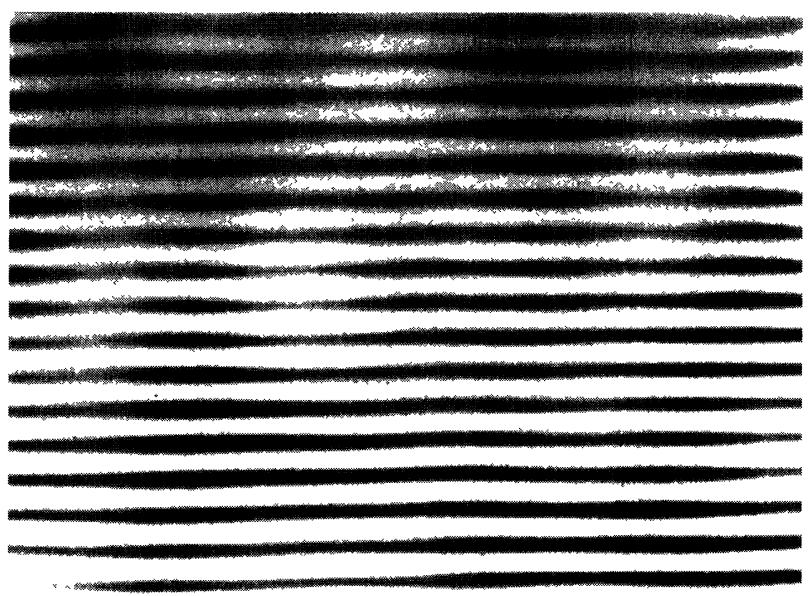

Fig. 5. - Filtered image, with one pair of reflections corresponding to one set of (220) planes, of an undisturbed part of the GaAs lattice. 


\section{Conclusions}

As shown by processing of HRTEM micrographs, extended defects (vacancy and interstitial type dislocation loops and edge dislocations) form in damaged zones of as-implanted GaAs due to the clustering of point defects. The calculated density of these extended defects is of the order of $10^{13} \mathrm{~cm}^{-2}$. The experimental data on the behaviour of different point or/and extended defects in a zinc-blend structure are very scarce.

From the above reported results, we can conclude that, at the used implantation conditions, point defects created in the displacement cascades tend to cluster forming small DLs.

By the way one can observe that this effect shows some analogy with the behaviour of FCC metals like $\mathrm{Cu}$ and $\mathrm{Ni}$ under irradiation with $\mathrm{MeV}$ protons, neutrons and heavy ions [14, 15]. In this case, by combined TEM and computer simulation, it has been demonstrated that selfinterstitial atoms (SIA) are clustering efficiently in energetic collision cascades [16]. Computer simulations of the behaviour of SIA-type dislocation loops in FCC metals indicate that at least the smaller ones are rather mobile. Thermally activated changes of the Burgers vectors of these defect structures in crystallographic equivalent directions result in a zig-zag motion of the DLs in the crystal lattice [16].

In the present case related to a zinc-blend compound semiconductor, the above reported HRTEM observations and previous HVTEM investigations [17], indicate that the LPPLA effects should be connected with the extended defects (both V and SIA types) migration induced by the absorbed laser energy. In fact, the "solid phase epitaxy" occurring in the laser annealed specimens, is related (in the authors' opinion) to the bonds weakening of the defect atoms which are ionized during the laser energy absorption process [6]. The laser induced extended defects migration, results in their accumulation in "defects lines" (high density defects alignments) parallel to the (110) planes as it was shown by HVTEM [17]. It is worth to notice that all the above discussed data, concerning the type, the concentration and the restoration degree of extended lattice defects in GaAs, are related only to the behaviour of isolated defect zones embedded in an undisturbed matrix in the case of a low-dose implantation $\left(10^{14} \mathrm{~cm}^{-2}\right)$. In order to put in evidence the quality of the restored structure treated with the LPPLA, the similarity between the filtered image from the undisturbed matrix region of as-implanted samples (Fig. 5) and that from a defect zone after the LPPLA treatment (Fig. 3) must be underlined. The undisturbed matrix shown in Figure 5 puts in evidence, as a peculiarity of low-dose ion-bombarded structures, the inhomogeneity of the induced damage. The last one is the indispensable condition for the LPPLA and the consequent epitaxy.

In the case of overlapping defect zones, the situation is much more complicated and will not be discussed in this paper.

The calculated temperature $T$ of the GaAs surface during LPPLA [8], does not exceed $700 \mathrm{~K}$. In this condition, the behavior of the LPPLA GaAs samples seems homologous to the behavior shown in the non-equilibrium-phase-diagram for Ni proposed by Jaeger et al. [14]. In this diagram, the appearance of periodic defect cluster arrangements parallel to (100) planes, in the case of neutrons, protons and heavy ions irradiation, is indicated to occur for $T=0.35 T_{\mathrm{m}}$, being $T_{\mathrm{m}}$ the melting temperature $[14,15]$.

As an open question remains the exact modelling of the LPPLA dynamical effects in the III-V compound semiconductor. Furthermore it is interesting to note that a standard thermal annealing of GaAs, at temperatures at which the LPPLA provokes an effective rearrangement of the defect clusters, has only a very restricted influence on the defect structures of ion implanted GaAs [18]. 


\section{Acknowledgements}

The investigations shown in the present paper have been carried out within the frames of the scientific agreement between the Italian CNR and the Bulgarian Academy of Sciences.

\section{References}

[1] Graf V., Heuberger W., Nucl. Instr. Meth. B 19/20 (1987) 388.

[2] Kalitzova M., Pashov N., Vitali G. and Rossi M., Mat. Res. Soc. Symp. Proc. 128 (1989) 683.

[3] Jager W., Trinkaus H., J. Nucl. Mater 205 (1993) 349.

[4] Vitali G., Rossi M., Karpuzov D., Budinov H., Kalitzova M., Katardjiev I., Nucl. Instr. Meth. Res. Phys. $B$ 59/60 (19S1) 1077.

[5] Vitali G., Jpn. J. Appl. Phys. 31 (1992) 2049.

[6] Vitali G., Pizzuto C., Rossi M., Zollo G., Karpuzov D., Kalitzova M., Jpn. J. Appl. Phys. 33 (1994) 2762.

[7] De Jong A.F., Coene W., Van Dyck D., Ultramicrosc. 27 (1989) 53.

[8] Zollo G., Palumbo L., Rossi M., Vitali G., Appl. Phys. A 56(1993) 409.

[9] Kalitzova M., Karpuzov D., Pashov N., Vitali G., Rossi M. and Scholtz R., Nucl. Instr. Meth. Phys. Res. B80/81 (1993) 647.

[10] Jones K.S., Rozgonyi G.A., Rapid Thermal Processing Science and Technology (Academic Press, 1993) p. 123.

[11] Jager W., Trinkaus H., J. Nucl. Mat. 205 (1993) 394.

[12] Von Guerard B., Grasse D. and Peisl J., Phys. Rev. Lett. 44 (1980) 262.

[13] Rauch R., Peisl J., Schmalzbauer A. and Wallner G., J. Nucl. Mater. 168 (1989) 101.

[14] Jager W., Ehrhart P., Shilling W., Solid State Phenomena 3/4 (1988) 279.

[15] Trinkaus H., Jager W., IFF Bulletin 41 (1992) 2.

[16] Jager W., Trinkaus H., Mater. Sci. Forum 123-125 (1993) 661.

[17] Pashov N., Kalitzova M., Vitali G., Rossi M. and Baither D., Jpn. J. Appl. Phys. 32 (1993) 2597.

[18] Morgen D.V., Eissen F.H., in Gallium Arsenide, Materials, Devices and Circuits (John Wiley \& Sons, 1985) p. 163. 